\title{
DIV-CURL TYPE THEOREMS ON LIPSCHITZ DOMAINS
}

\section{ZENGJIAN LOU}

For Lipschitz domains of $\mathbb{R}^{n}$ we prove div-curl type theorems, which are extensions to domains of the Div-Curl Theorem on $\mathbb{R}^{n}$ by Coifman, Lions, Meyer and Semmes. Applying the div-curl type theorems we give decompositions of Hardy spaces on domains.

\section{INTRODUCTION}

In [4] two Hardy spaces are defined on domains $\Omega$ of $\mathbb{R}^{n}$, one which is reasonably speaking the largest, and the other which in a sense is the smallest. The largest, $\mathcal{H}_{r}^{1}(\Omega)$, arises by restricting to $\Omega$ arbitrary elements of $\mathcal{H}^{1}\left(\mathbb{R}^{n}\right)$. The other, $\mathcal{H}_{z}^{1}(\Omega)$, arises by restricting to $\Omega$ elements of $\mathcal{H}^{1}\left(\mathbb{R}^{n}\right)$ which are zero outside $\bar{\Omega}$. Norms on these spaces are defined as following

$$
\|f\|_{\mathcal{H}_{r}^{1}(\Omega)}=\inf \|F\|_{\mathcal{H}^{1}\left(\mathbb{R}^{n}\right)}
$$

the infimum being taken over all functions $F \in \mathcal{H}^{1}\left(\mathbb{R}^{n}\right)$ such that $\left.F\right|_{\Omega}=f$,

$$
\|f\|_{\mathcal{H}_{z}^{1}(\Omega)}=\|F\|_{\mathcal{H}^{1}\left(\mathbb{R}^{n}\right)}
$$

where $F$ is the zero extension of $f$ to $\mathbb{R}^{n}$.

From [2], the dual of $\mathcal{H}_{z}^{1}(\Omega)$ is $\mathrm{BMO}_{r}(\Omega)$, a space of locally integrable functions with

$$
\|f\|_{\mathrm{BMO}_{r}(\Omega)}=\sup _{Q \subset \Omega}\left(\frac{1}{|Q|} \int_{Q}\left|f(x)-f_{Q}\right|^{2} d x\right)^{1 / 2}<\infty,
$$

where $f_{Q}=1 /|Q| \int_{Q} f(x) d x$, and the supremum is taken over all cubes $Q$ in the domain $\Omega$. The dual of $\mathcal{H}_{r}^{1}(\Omega)$ is $\mathrm{BMO}_{z}(\Omega)$, the space of all functions in $\mathrm{BMO}\left(\mathbb{R}^{n}\right)$ supported in $\bar{\Omega}$, equipped with the norm $\|f\|_{\mathrm{BMO}_{z}(\Omega)}=\|f\|_{\mathrm{BMO}\left(\mathbb{R}^{n}\right)}$.

Received 24th January, 2005

This work is supported by NNSF of China (Grant No.10371069), NSF of Guangdong Province (Grant No.032038) and SRF for ROCS, State Education Ministry. This paper was done when the author visited the Centre for Mathematics and its Applications (CMA) of Mathematical Sciences Institute at the Australian National University. The author would like to thank Professor Alan $\mathbf{M}^{c}$ Intosh for helpful discussions and for supporting his visit to CMA in September of 2003. He also likes to thank CMA for hospitality during the visit.

Copyright Clearance Centre, Inc. Serial-fee code: 0004-9727/05 \$A2.00+0.00. 
Let $\Omega$ denote a Lipschitz domain - an assumption which is enough to ensure the existence of a bounded extension map from $\mathrm{BMO}_{r}(\Omega)$ to $\mathrm{BMO}\left(\mathbb{R}^{n}\right)([6])$. We use $H(\Omega)^{n}:=H\left(\Omega, \mathbb{R}^{n}\right.$ ) to denote a space of functions $f: \Omega \rightarrow \mathbb{R}^{n}$ (when $n=1$, write $H(\Omega)^{1}$ as $\left.H(\Omega)\right)$. For simplicity we introduce the following spaces

$$
\begin{aligned}
& L_{\text {div }}^{2}(\Omega)^{n}=\left\{f \in L^{2}(\Omega)^{n}: \operatorname{div} f=0,\left.\nu \cdot f\right|_{\partial \Omega}=0,\|f\|_{L^{2}(\Omega)^{n}} \leqslant 1\right\} \\
& L_{\text {curl }}^{2}(\Omega)^{n}=\left\{f \in L^{2}(\Omega)^{n}: \operatorname{curl} f=0, \nu \times\left. f\right|_{\partial \Omega}=0,\|f\|_{L^{2}(\Omega)^{n}} \leqslant 1\right\}
\end{aligned}
$$

where $\nu$ denotes the outward unit normal vector. When $\Omega=\mathbb{R}^{n}$

$$
\begin{aligned}
& L_{\text {div }}^{2}\left(\mathbb{R}^{n}\right)^{n}=\left\{f \in L^{2}\left(\mathbb{R}^{n}\right)^{n}: \operatorname{div} f=0,\|f\|_{L^{2}\left(\mathbb{R}^{n}\right)^{n}} \leqslant 1\right\} \\
& L_{\text {curl }}^{2}\left(\mathbb{R}^{n}\right)^{n}=\left\{f \in L^{2}\left(\mathbb{R}^{n}\right)^{n}: \operatorname{curl} f=0,\|f\|_{L^{2}\left(\mathbb{R}^{n}\right)^{n}} \leqslant 1\right\}
\end{aligned}
$$

In [5, Theorems II.1 and III.2], among other results, Coifman, Lions, Meyer and Semmes established the following theorems.

ThEOREM CLMS1. Let $1<p, q<\infty, 1 / p+1 / q=1, E \in L^{p}\left(\mathbb{R}^{n}\right)^{n}, \operatorname{div} E=0$, $F \in L^{q}\left(\mathbb{R}^{n}\right)^{n}$, curl $F=0$. Then $E \cdot F \in \mathcal{H}^{1}\left(\mathbb{R}^{n}\right)$ and

$$
\|E \cdot F\|_{\mathcal{H}^{1}\left(\mathbb{R}^{n}\right)} \leqslant C\|E\|_{L^{p}\left(\mathbb{R}^{n}\right)^{n}}\|F\|_{L^{q}\left(\mathbb{R}^{n}\right)^{n}}
$$

for a constant $C$ depending only on the dimension $n$.

THEOREM CLMS2. For $b \in \mathrm{BMO}\left(\mathbb{R}^{n}\right)$

$$
\|b\|_{\mathrm{BMO}\left(\mathbb{R}^{n}\right)} \approx \sup _{E, F} \int_{\mathbb{R}^{n}} b E \cdot F d x
$$

where the supremum is taken over all $E \in L^{2}\left(\mathbb{R}^{n}\right)^{n}, F \in L^{2}\left(\mathbb{R}^{n}\right)^{n}$ with $\operatorname{div} E=0$, curl $F=0$ and $\|E\|_{L^{2}\left(\mathbb{R}^{n}\right)^{n}} \leqslant 1,\|F\|_{L^{2}\left(\mathbb{R}^{n}\right)^{n}} \leqslant 1$, and the implicit constants in (1.2) depend only on $n$.

A natural question to ask is: under what conditions on domains $\Omega$ does the equivalence (1.2) hold on $\Omega$ ? As a main theorem of this paper, we solve this problem for Lipschitz domains in $\mathbb{R}^{n}$.

THEOREM 1.1. Let $\Omega$ be a Lipschitz domain of $\mathbb{R}^{n}$.

(1) If $b \in \mathrm{BMO}_{r}(\Omega)$, then

$$
\|b\|_{\mathrm{BMO}_{r}(\Omega)} \approx \sup _{e, f} \int_{\Omega} b e \cdot f d x
$$

the supremum being taken over all $e \in L_{\text {div }}^{2}(\Omega)^{n}, f \in L_{\text {curl }}^{2}(\Omega)^{n}$.

(2) If $b \in \mathrm{BMO}_{z}(\Omega)$, then 


$$
\|b\|_{\mathrm{BMO}_{z}(\Omega)} \approx \sup _{e, f} \int_{\Omega} b e \cdot f d x
$$

the supremum being taken over all $e=\left.E\right|_{\Omega}, f=\left.F\right|_{\Omega}, E \in L_{\mathrm{div}}^{2}\left(\mathbb{R}^{n}\right)^{n}$, $F \in L_{\text {curl }}^{2}\left(\mathbb{R}^{n}\right)^{n}$. The implicit constants in (1.3) and (1.4) depend only on the domain $\Omega$ and the dimension $n$.

REMARK. Results for other BMO-type spaces, such as dual of divergence-free Hardy spaces, can be found in [8] and [9].

COROLlary 1.2 .

(1) A function $b \in \mathrm{BMO}_{r}(\Omega)$ if and only if there exists a constant $C$ such that $\int_{\Omega} b e \cdot f d x \leqslant C$ for all $e \in L_{\text {div }}^{2}(\Omega)^{n}$ and $f \in L_{\text {curl }}^{2}(\Omega)^{n}$.

(2) A function $b \in \mathrm{BMO}_{z}(\Omega)$ if and only if there exists a constant $C$ such that $\int_{\Omega} b e \cdot f d x \leqslant C$ for all $e=\left.E\right|_{\Omega}$ and $f=\left.F\right|_{\Omega}$ with $E \in L_{\mathrm{div}}^{2}\left(\mathbb{R}^{n}\right)^{n}$, $F \in L_{\text {curl }}^{2}\left(\mathbb{R}^{n}\right)^{n}$.

Here and afterwards, unless otherwise specified, $C$ denotes a constant depending only on the domain $\Omega$ and the dimension $n$. Such $C$ may differ at different occurrences.

Applying Theorem 1.1 we have the following theorem which gives decompositions of $\mathcal{H}_{z}^{1}(\Omega)$ and $\mathcal{H}_{r}^{1}(\Omega)$ into quantities of forms " $e \cdot f$ ".

\section{THEOREM 1.3 .}

(1) Any function $u \in \mathcal{H}_{z}^{1}(\Omega)$ can be written as

$$
u=\sum_{k=1}^{\infty} \lambda_{k} e_{k} \cdot f_{k}
$$

where $e_{k} \in L_{\mathrm{div}}^{2}(\Omega)^{n}, f_{k} \in L_{\text {curl }}^{2}(\Omega)^{n}$ and $\sum_{k=1}^{\infty}\left|\lambda_{k}\right|<\infty$.

(2) Any function $u \in \mathcal{H}_{r}^{1}(\Omega)$ can be written as

$$
u=\sum_{k=1}^{\infty} \lambda_{k} e_{k} \cdot f_{k}
$$

where $e_{k}=\left.E_{k}\right|_{\Omega}, f_{k}=\left.F_{k}\right|_{\Omega}, E_{k} \in L_{\text {div }}^{2}\left(\mathbb{R}^{n}\right)^{n}, F_{k} \in L_{\text {curl }}^{2}\left(\mathbb{R}^{n}\right)^{n}$ and $\sum_{k=1}^{\infty}\left|\lambda_{k}\right|<\infty$. 


\section{PROOF OF THEOREM 1.1}

To prove Theorem 1.1, we need the following lemmas.

Lemma 2.1. ([6, Theorem 1].) Let $b \in \mathrm{BMO}_{r}(\Omega)$. Then there exists $B \in \mathrm{BMO}\left(\mathbb{R}^{n}\right)$ such that

$$
b=\left.B\right|_{\Omega}
$$

and

$$
\|B\|_{\mathrm{BMO}\left(\mathbb{R}^{n}\right)} \leqslant C\|b\|_{\mathrm{BMO}(\Omega)}
$$

Lemma 2.2. ([7, Theorem 3.1].) Let $b$ be a locally integrable function on $\Omega$. Then

$$
\|b\|_{\mathrm{BMO}_{r}(\Omega)} \approx\|b\|_{\mathrm{BMO}^{H}(\Omega)}
$$

where

$$
\|b\|_{\mathrm{BMO}^{H}(\Omega)}=\sup _{Q}\left(\frac{1}{|Q|} \int_{Q}\left|b-b_{Q}\right|^{2} d x\right)^{1 / 2}
$$

the supremum being taken over all cubes $Q$ with $2 Q \subset \Omega$, the implicit constants in (2.2) depend only on $\Omega$ and $n$.

LEMMA 2.3. For $b \in L_{l o c}^{2}(\Omega)$

$$
\|b\|_{\mathrm{BMO}^{H}(\Omega)} \leqslant C \sup _{e, f} \int_{\Omega} b e \cdot f d x
$$

the supremum being taken over all $e \in L_{\text {div }}^{2}(\Omega)^{n}$ and $f \in L_{\text {curl }}^{2}(\Omega)^{n}$.

The proof of Lemma 2.3 is given in the last section.

Proof of THEOREM 1.1: (1) Let $B \in \mathrm{BMO}\left(\mathbb{R}^{n}\right)$ be an extension of $b \in \mathrm{BMO}_{r}(\Omega)$ such that $b=\left.B\right|_{\Omega}$ and (2.1) holds. For $e \in L_{\text {div }}^{2}(\Omega)^{n}, f \in L_{\text {curl }}^{2}(\Omega)^{n}$, define

$$
\begin{aligned}
& E= \begin{cases}e & \text { in } \Omega ; \\
0 & \text { in } \mathbb{R}^{n} \backslash \Omega,\end{cases} \\
& F= \begin{cases}f & \text { in } \Omega ; \\
0 & \text { in } \mathbb{R}^{n} \backslash \Omega .\end{cases}
\end{aligned}
$$

Since $\operatorname{div} e=0$ on $\Omega$ and $\left.e \cdot \nu\right|_{\partial \Omega}=0$, it is easy to show that $\operatorname{div} E=0$ on $\mathbb{R}^{n}$. So $E \in L_{\text {div }}^{2}\left(\mathbb{R}^{n}\right)^{n}$. Similarly, curl $f=0$ on $\Omega$ and $f \times\left.\nu\right|_{\partial \Omega}=0$ imply that $\operatorname{curl} F=0$ 
on $\mathbb{R}^{n}$. Therefore $F \in L_{\text {curl }}^{2}\left(\mathbb{R}^{n}\right)^{n}$. By duality $\mathcal{H}^{1}\left(\mathbb{R}^{n}\right)^{*}=\operatorname{BMO}\left(\mathbb{R}^{n}\right)$, Lemma 2.1 and (1.1), we have

$$
\begin{aligned}
\int_{\Omega} b e \cdot f d x & =\int_{\mathbf{R}^{n}} B E \cdot F d x \leqslant\|B\|_{\mathrm{BMO}\left(\mathbb{R}^{n}\right)}\|E \cdot F\|_{\mathcal{H}^{1}\left(\mathbf{R}^{n}\right)} \\
& \leqslant C\|b\|_{\mathrm{BMO}_{r}(\Omega)}\|E\|_{L^{2}\left(\mathbf{R}^{n}\right)^{n}}\|F\|_{L^{2}\left(\mathbf{R}^{n}\right)^{n}} \\
& =C\|b\|_{\mathrm{BMO}_{\mathrm{r}}(\Omega)}\|e\|_{L^{2}(\Omega)^{n}}\|f\|_{L^{2}(\Omega)^{n}} \leqslant C\|b\|_{\mathrm{BMO}_{r}(\Omega)} .
\end{aligned}
$$

The proof of the reversed inequality in (1.3) follows from (2.2) and (2.3).

(2) Let $b \in \mathrm{BMO}_{z}(\Omega)$ and $B$ be its zero extension to $\mathbb{R}^{n}$. Then $B \in \mathrm{BMO}\left(\mathbb{R}^{n}\right)$ and $\|B\|_{\mathrm{BMO}\left(\mathbb{R}^{n}\right)}=\|b\|_{\mathrm{BMO}_{z}(\Omega)}$. Using (1.1) again,

$$
\begin{aligned}
\int_{\Omega} b e \cdot f d x=\int_{\mathbb{R}^{n}} B E \cdot F d x & \leqslant\|B\|_{\mathrm{BMO}\left(\mathbb{R}^{n}\right)}\|E \cdot F\|_{\mathcal{H}^{1}\left(\mathbb{R}^{n}\right)} \\
& \leqslant C\|b\|_{\mathrm{BMO}_{z}(\Omega)}\|E\|_{L^{2}\left(\mathbb{R}^{n}\right)^{n}}\|F\|_{L^{2}\left(\mathbb{R}^{n}\right)^{n}} \\
& \leqslant C\|b\|_{\mathrm{BMO}_{z}(\Omega)}
\end{aligned}
$$

for all $e=\left.E\right|_{\Omega}, f=\left.F\right|_{\Omega}, E \in L_{\text {div }}^{2}\left(\mathbb{R}^{n}\right)^{n}, F \in L_{\text {curl }}^{2}\left(\mathbb{R}^{n}\right)^{n}$.

For the converse, let $b \in \mathrm{BMO}_{z}(\Omega)$ and define $B$ as above. Applying (1.2) yields

$$
\begin{aligned}
\|b\|_{\mathrm{BMO}}(\Omega) & =\|B\|_{\mathrm{BMO}\left(\mathbb{R}^{n}\right)} \leqslant C \sup _{E \in L_{\mathrm{div}}^{2}, F \in L_{\text {curl }}^{2}} \int_{\mathbb{R}^{n}} B E \cdot F d x \\
& =C \sup _{e=\left.E\right|_{\Omega}, f=\left.F\right|_{\Omega}, E \in L_{\mathrm{div}}^{2}, F \in L_{\text {curl }}^{2}} \int_{\Omega} b e \cdot f d x .
\end{aligned}
$$

Theorem 1.1 is proved.

\section{Proof of Theorem 1.3}

The proof of Theorem 1.3 relies on Theorem 1.1 and the following facts from functional analysis which can be found in [5, Lemmas III.1, III.2].

Lemma 3.1. Let $V$ be a bounded subset of a normed vector space $X$. We assume that $\bar{V}$ (closure of $V$ for the norm of $X$ ) contains the unit ball (centred at 0 ) of $X$. Then, any $x$ in that ball can be written as

$$
x=\sum_{j=0}^{\infty} \frac{1}{2^{j}} y_{j},
$$

where $y_{j} \in V$ for all $j \geqslant 0$.

LEMMA 3.2. Let $V$ be a bounded symmetric $(x \in V \Rightarrow-x \in V)$ subset of a normed vector space $X$. Then, the closed convex hull $\tilde{V}$ of $V$ (in $X$ ) contains a ball centred at 0 if and only if, for any $l \in X^{*}$,

$$
\|l\|_{X^{*}} \approx \sup _{x \in V}\langle l, x\rangle
$$


Proof of TheOREM 1.3: (1) Let $X=\mathcal{H}_{z}^{1}(\Omega)$ and

$$
V=\left\{e \cdot f: e \in L_{\text {div }}^{2}(\Omega)^{n}, f \in L_{\text {curl }}^{2}(\Omega)^{n}\right\}
$$

It is easy to check that $V$ is a bounded subset of $X$. In fact, for $e \in L_{\mathrm{div}}^{2}(\Omega)^{n}$, $f \in L_{\text {curl }}^{2}(\Omega)^{n}$, let $E$ and $F$ be their zero extensions to $\mathbb{R}^{n}$ respectively. Then $E \in L_{\text {div }}^{2}\left(\mathbb{R}^{n}\right)^{n}, F \in L_{\text {curl }}^{2}\left(\mathbb{R}^{n}\right)^{n}$. From Theorem CLMS1, $E \cdot F \in \mathcal{H}^{1}\left(\mathbb{R}^{n}\right)$ and

$$
\|E \cdot F\|_{\mathcal{H}^{1}\left(\mathbb{R}^{n}\right)} \leqslant C\|E\|_{L^{2}\left(\mathbb{R}^{n}\right)^{n}}\|F\|_{L^{2}\left(\mathbb{R}^{n}\right)^{n}} \leqslant C .
$$

Therefore $e \cdot f \in \mathcal{H}_{z}^{1}(\Omega)$ with $\|e \cdot f\|_{\mathcal{H}_{z}^{1}(\Omega)} \leqslant C$. Applying Theorem $1.1(1)$ and Lemmas 3.1 and 3.2, we have the decomposition of Theorem $1.3(1)$.

(2) Let $X=\mathcal{H}_{r}^{1}(\Omega)$ and

$$
V=\left\{e \cdot f: e=\left.E\right|_{\Omega}, f=\left.F\right|_{\Omega}, E \in L_{\mathrm{div}}^{2}\left(\mathbb{R}^{n}\right)^{n}, F \in L_{\mathrm{curl}}^{2}\left(\mathbb{R}^{n}\right)^{n}\right\}
$$

Similar to the case (1), we have $e \cdot f \in \mathcal{H}_{r}^{1}(\Omega)$ with

$$
\|e \cdot f\|_{\mathcal{H}_{r}^{1}(\Omega)}=\inf _{e \cdot f=\left.G\right|_{\Omega}, G \in \mathcal{H}^{1}\left(\mathbb{R}^{n}\right)}\|G\|_{\mathcal{H}^{1}\left(\mathbb{R}^{n}\right)} \leqslant\|E \cdot F\|_{\mathcal{H}^{1}\left(\mathbb{R}^{n}\right)} \leqslant C
$$

for $e \cdot f \in V$. Using Theorem 1.1 (2) and those two lemmas again we finish the proof of Theorem 1.3.

\section{Proof of LeMMA 2.3}

To prove Lemma 2.3 we need the following result due to Nečas (see [10, Lemma 7.1, Chapter 3]). In Lemma 4.1, $W_{0}^{1,2}(\Omega)^{n}$ denotes the closure of $C_{0}^{\infty}(\Omega)^{n}$ in the Sobolev space $W^{1,2}(\Omega)^{n}$ and $\nabla \varphi=\left(\left(\partial \varphi_{i}\right) /\left(\partial x_{j}\right)\right)_{n \times n}$ a $n \times n$ matrix (see [1] for Sobolev spaces).

LEMMA 4.1. Let $\Omega$ be a Lipschitz domain in $\mathbb{R}^{n}$. If $f \in L^{2}(\Omega)$ has zero integral, then there exists $\varphi \in W_{0}^{1,2}(\Omega)^{n}$ such that

$$
f=\operatorname{div} \varphi
$$

and

$$
\|\nabla \varphi\|_{L^{2}(\Omega)^{n \times n}} \leqslant C\|f\|_{L^{2}(\Omega)}
$$

Corollary 4.2. Let $Q$ be a cube in $\mathbb{R}^{n}$. If $f \in L^{2}(Q)$ has zero integral, then there exists $\varphi \in W_{0}^{1,2}(Q)^{n}$ such that $f=\operatorname{div} \varphi$ and

$$
\|\nabla \varphi\|_{L^{2}(Q)^{n \times n}} \leqslant C_{0}\|f\|_{L^{2}(Q)}
$$


for a constant $C_{0}$ independent of $Q$.

Proof of Lemma 2.3: Suppose $b \in L_{\text {loc }}^{2}(\Omega)$. We shall show that for all cubes $Q$ with $2 Q \subset \Omega$ there exists $e \in L_{\text {div }}^{2}(\Omega)^{n}$ and $f \in L_{\text {curl }}^{2}(\Omega)^{n}$ such that

$$
\left(\frac{1}{|Q|} \int_{Q}\left|b-b_{Q}\right|^{2} d x\right)^{1 / 2} \leqslant C\left|\int_{\Omega} b e \cdot f d x\right| .
$$

Let $h=b-b_{Q}$, then $h \in L^{2}(Q)$ with $\int_{Q} h d x=0$. From Corollary 4.2, there exists $\varphi:=\left(\varphi_{1}, \ldots, \varphi_{n}\right) \in W_{0}^{1,2}(Q)^{n}$ such that $h=\operatorname{div} \varphi$ and

$$
\|\nabla \varphi\|_{L^{2}(Q)^{n \times n}} \leqslant C_{0}\|h\|_{L^{2}(Q)}
$$

where $C_{0}$ is independent of $Q$. So

$$
\begin{aligned}
\|h\|_{L^{2}(Q)}^{2} & =\int_{Q} h \sum_{i=1}^{n} \frac{\partial \varphi_{i}}{\partial x_{i}} d x \leqslant n \max _{1 \leqslant i \leqslant n}\left|\int_{Q} h \frac{\partial \varphi_{i}}{\partial x_{i}} d x\right| \\
& =n\left|\int_{Q} h \frac{\partial \varphi_{i_{0}}}{\partial x_{i_{0}}} d x\right|
\end{aligned}
$$

for some choice of $i_{0}\left(i_{0}=1, \ldots, n\right)$. Assuming without loss of generality that $i_{0}=1$ in (4.3). To prove (4.1), it is sufficient to show that

$$
\left|\int_{Q} h\|h\|_{L^{2}(Q)}^{-1} \frac{\partial \varphi_{1}}{\partial x_{1}} d x\right| \leqslant C|Q|^{1 / 2}\left|\int_{Q} h e \cdot f d x\right|
$$

We next construct $e$ and $f$. Define

$$
f=\left(-\frac{\partial \varphi_{1}}{\partial x_{i}}, 0, \ldots, 0, \frac{\partial \varphi_{1}}{\partial x_{1}}, 0, \ldots, 0\right) C_{0}^{-1}\|h\|_{L^{2}(Q)}^{-1}
$$

where $\left(\partial \varphi_{1}\right) /\left(\partial x_{1}\right)$ is the i-th component of $f$. Then $f \in L^{2}(Q)^{n}$ with $\operatorname{div} f=0$ and $\|f\|_{L^{2}(Q)^{n}} \leqslant 1$ by $(4.2)$.

Let $\psi_{0} \in C_{0}^{\infty}\left(\mathbb{R}^{n}\right)$ such that

$$
\psi_{0}= \begin{cases}1 & \text { on }[-1,1]^{n} \\ 0 & \text { outside }[-2,2]^{n}\end{cases}
$$

Define

$$
e=\gamma C_{0}|Q|^{-1 / 2} \nabla\left(\left(x_{i}-x_{i}^{0}\right) \psi_{Q}(x)\right), \quad 1 \leqslant i \leqslant n,
$$


where $\psi_{Q}(x)=\psi_{0}\left(\left(x-x^{0}\right) /(l(Q) / 2)\right), x^{0}=\left(x_{1}^{0}, \ldots, x_{n}^{0}\right)$ and $l(Q)$ denote the centre and the side-length of the cube $Q, \gamma>0$ is a normalisation constant (independent of $x^{0}$ and $l(Q))$ so that $\|e\|_{L^{2}(\Omega)^{n}} \leqslant 1$. It is obvious that $e \in C_{0}^{\infty}(2 Q)$ and $e=\gamma C_{0}|Q|^{-1 / 2} \varepsilon_{i}$ on $Q$, where $\varepsilon_{i}=(0, \ldots, 0,1,0, \ldots, 0), 1$ is the $i$-th component of $\varepsilon_{i}$. From the construction of $e$ and $f$, we get

$$
e \cdot f=\gamma|Q|^{-1 / 2}\|h\|_{L^{2}(Q)}^{-1} \frac{\partial \varphi_{1}}{\partial x_{1}} \quad \text { on } \quad Q
$$

and (4.4) is proved.

Note. It should be added that at the time the paper was finished, the author was unfortunately unaware of a similar but unpublished work [3] (with different proof). Thanks go to Galia Dafni (Department of Mathematics \& Statistics, Concordia University, Canada) for informing us her paper with Chang and Sadosky.

\section{REFERENCES}

[1] R.A. Adams, Sobolev spaces (Academic Press, New York, 1975).

[2] D.C. Chang, 'The dual of Hardy spaces on a domain in $\mathbb{R}^{n}$, Forum Math. 6 (1994), 65-81.

[3] D.C. Chang, G. Dafni and C. Sadosky, 'A div-curl lemma in BMO on a domain' (to appear).

[4] D.C. Chang, S.G. Krantz and E.M. Stein, ' $\mathcal{H}^{p}$ theory on a smooth domain in $\mathbb{R}^{n}$ and elliptic boundary value problems', J. Funct. Anal. 114 (1993), 286-347.

[5] R. Coifman, P.L. Lions, Y. Meyer and S. Semmes, 'Compensated compactness and Hardy spaces', J. Math. Pures Appl. 72 (1993), 247-286.

[6] P.W. Jones, 'Extension theorems for BMO', Indiana Univ. Math. J. 29 (1980), 41-66.

[7] Z. Lou, 'Jacobian on Lipsctitz domains of $\mathbb{R}^{2}$ ', Proc. Centre Math. Appl. Austral. Nat. Univ. 41 (2003), 96-109.

[8] Z. Lou and A. Mc Intosh, 'Hardy spaces of exact forms on $\mathbb{R}^{n}$ ', Trans. Amer. Math. Soc. 357 (2005), 1469-1496.

[9] Z. Lou and A. $M^{c}$ Intosh, 'Hardy spaces of exact forms on Lipschitz domains in $\mathbb{R}^{n}$, Indiana Univ. Math. J. 54 (2004), 581-609.

[10] J. Nečas, Les méthodes directes en théorie des équations elliptiques, (Masson et Cie Paris, Editors) (Academia, Editeurs, Prague, 1967).

Department of Mathematics

Shantou University

Shantou

Guangdong 515063

Peoples Repbulic of China

e-mail: zjlou@stu.edu.cn 